\title{
Investigation of vibration characteristics for bladed-disks with dry friction nonlinearity
}

\author{
Tianyuan Liu, ${ }^{1, a}$, Yonghui Xie ${ }^{1}$ and Di Zhang ${ }^{1}$ \\ ${ }^{1}$ MOE Key Laboratory of Thermo-Fluid Science and Engineering, School of Energy and Power Engineering, Xi'an Jiaotong University, \\ 710049 Xi'an, China
}

\begin{abstract}
This paper focuses on the vibration characteristics of the bladed-disk subjected to the dry friction damping under periodic excitation. Firstly, the multi-harmonic equation basing on the frequency-domain analysis is established to predicate the steady response of the bladed-disk. Then, the algorithm to solve the nonlinear multi-harmonic balance equation is given step by step. In the numerical simulation, a simplified lumped parameter model of a turbine bladeddisk as well as the elastic Coulomb friction model between the root and disk contacting surfaces are applied. The normal load level of the friction interfaces, which are the focal points of the bladed-disk design, is analysed for the nonlinear vibration characteristics of the blades. The results show that vibration response of bladed-disk is affected significantly by normal load, and there exists an optimal value of the normal load under the operating condition of the turbomachinery, which can provide a qualitative assessment for the design practice of friction dampers.
\end{abstract}

\section{Introduction}

In operating conditions, the turbomachinery undergoes many sources of excitation, making vibration mitigation a challenge of great interest in bladed-disk design. Friction dampers such as snubbers, shrouds and under-platform dampers, which turn out to be the most effective method to reduce vibration, are widely used in modern turbomachinery.

Due to the strong nonlinearity of the friction dampers, it is difficult to predict the response of the bladed disks under periodic excitation. Especially, it is a big problem to design the dampers to reduce the vibration of the structure. For the approximate linear model widely adopted in industrial, $\mathrm{Wu}$ and Yuan [1] applied the equivalent linearization of the stiffness and damping coefficients of the friction dampers, which can be regarded as single harmonic method. However, this method has been proved with low accuracy in hazardous conditions. Recently, analysis of the vibrations of bladed discs with friction dampers is usually performed in the frequency-domain owing to the high computational efficiency of this approach. Petrov [2] derived the analytical formulation for the vectors of contact forces and the stiffness matrix of the non-linear friction contact interface based on multi-harmonic vibrations in the frequency-domain, and developed this method for highfidelity models of different bladed disks [3, 4]. Laxalde et al. [5] proposed an alternating frequency-time domain method for the calculation of implicit non-linear forces. Joannin et al. [6] investigated the phenomenology of turbomachinery bladed disk subjected to dry friction nonlinearities by means of a lumped-parameter model. These investigations expand the scope of multi-harmonic methods in the design of bladed-disk.

This paper is organized as follows: 1 . The dynamic equation for bladed disk is established in accordance with the multi-harmonic balance method; 2 . The algorithm to solve the nonlinear equation is given step by step; 3 . The elastic Coulomb friction model with variable norm load is performed to the root-disk friction interface of the bladed disk. At last, a simplified lumped parameter model is applied in the numerical simulation to investigate how the contact norm load of the dampers impact on the response of the bladed-disks.

\section{Multi-harmonic balance equations}

The dynamic equation for forced vibration of a bladeddisk system with nonlinear interactions can be can be written in the form:

$$
\boldsymbol{K x}+\boldsymbol{C} \dot{\boldsymbol{x}}+\boldsymbol{M} \ddot{\boldsymbol{x}}=\boldsymbol{f n}(\boldsymbol{x}, \dot{\boldsymbol{x}}, \ddot{\boldsymbol{x}})+f \boldsymbol{e}(t)
$$

Where, $\boldsymbol{x}, \dot{\boldsymbol{x}}, \ddot{\boldsymbol{x}}$ are vectors of displacements, velocities and acceleration for all degrees of freedom (DOFs) in the bladed-disk structure, respectively. $\boldsymbol{M}, \boldsymbol{C}, \boldsymbol{K}$ are structural stiffness, damping and mass matrices. $\boldsymbol{f e}(t)$ is a vector of excitation forces and $\boldsymbol{f} \boldsymbol{n}(\boldsymbol{x}, \dot{\boldsymbol{x}}, \ddot{\boldsymbol{x}})$ is the vector of nonlinear forces which, for a general case considered here, can be explicitly dependent on $\boldsymbol{x}, \dot{\boldsymbol{x}}, \ddot{\boldsymbol{x}}$. It should be noticed that the excitation forces of the bladed disk in equation (1) are usually considered as the periodic forces, which satisfy $f e(t)=f e(t+T)$, where vibration period $T$ can be written as $T=2 \pi / \omega$.

\footnotetext{
$\overline{{ }^{a} \text { Corresponding author: tianyuannn } @ 163 . c o m}$
} 
Then time variation of displacements $\boldsymbol{x}$, the nonlinear forces or the periodic excitation forces $f$ for the steadystate periodic regimes by a restricted Fourier series:

$$
\begin{gathered}
x(t)=X_{0}+\sum_{i=1}^{N h}\left(X_{i}^{c} \cos \omega_{i} t+X_{i}^{s} \sin \omega_{i} t\right) \\
f(t)=f_{0}+\sum_{i=1}^{N h}\left(f_{i}^{c} \cos \omega_{i} t+f_{i}^{s} \sin \omega_{i} t\right)
\end{gathered}
$$

For multi-harmonic equations, the frequencies $\omega_{i}$ of the harmonic components can be expressed as $i \omega$, and $N h$ is the number of harmonic. Let $\boldsymbol{B}$ denotes the transformation matrix and $\boldsymbol{X}, \boldsymbol{F}$ denote the vectors of harmonic coefficients for displacements, nonlinear forces and excitation forces respectively. The term $\boldsymbol{X}, \boldsymbol{F}$ in the frequency-domain can be expressed as:

$$
\begin{aligned}
& \boldsymbol{x}=\boldsymbol{B} \boldsymbol{X}=\boldsymbol{B}\left\{\boldsymbol{X}_{0}, \boldsymbol{X}_{c}^{1}, \cdots, \boldsymbol{X}_{s}^{N h}\right\}^{\mathrm{T}} \\
& \boldsymbol{f}=\boldsymbol{B} \boldsymbol{F}=\boldsymbol{B}\left\{\boldsymbol{F}_{0}, \boldsymbol{F}_{c}^{1}, \cdots, \boldsymbol{F}_{s}^{N h}\right\}^{\boldsymbol{T}}
\end{aligned}
$$

The multi-harmonic balance procedure of the timedomain Eq. (1) gives the following nonlinear equation with respect to vector $\mathrm{X}$.

$$
\begin{gathered}
A X=F n+F e \\
A=\bar{K}+\bar{C} T_{C}+\bar{M} T_{M}
\end{gathered}
$$

Where $\quad \overline{\boldsymbol{K}}=\operatorname{diag}(\boldsymbol{K}, \cdots, \boldsymbol{K}) \quad, \quad \overline{\boldsymbol{C}}=\operatorname{diag}(\boldsymbol{C}, \cdots, \boldsymbol{C}) \quad$, $\overline{\boldsymbol{M}}=\operatorname{diag}(\boldsymbol{M}, \cdots, \boldsymbol{M})$ and the matrices $\boldsymbol{T}_{\boldsymbol{C}}$ and $\boldsymbol{T}_{\boldsymbol{M}}$ in Eq.

(7) can be written as:

$$
\begin{gathered}
\boldsymbol{T}_{C}=\operatorname{diag}\left(0, \omega_{1}\left[\begin{array}{cc}
\boldsymbol{0} & \boldsymbol{I} \\
-\boldsymbol{I} & \boldsymbol{0}
\end{array}\right], \cdots, \omega_{N h}\left[\begin{array}{cc}
\boldsymbol{0} & \boldsymbol{I} \\
-\boldsymbol{I} & \boldsymbol{0}
\end{array}\right]\right) \\
\boldsymbol{T}_{M}=\operatorname{diag}\left(0,-\omega_{1}^{2}\left[\begin{array}{cc}
\boldsymbol{I} & \boldsymbol{0} \\
\boldsymbol{0} & \boldsymbol{I}
\end{array}\right], \cdots,-\omega_{N h}{ }^{2}\left[\begin{array}{ll}
\boldsymbol{I} & \boldsymbol{0} \\
\boldsymbol{0} & \boldsymbol{I}
\end{array}\right]\right)
\end{gathered}
$$

\section{Arc-length solver for nonlinear equations}

The Newton-Raphson method is usually applied to solve the nonlinear equation Eq. (6), however, it always causes difficulty in convergence near the resonance frequency. In order to efficiently deal with the convergence problem, an arc-length continuation was used to trace the loci of the solutions [6]. An equation is required to supplement Eq. (6) :

$$
d s=\sqrt{\left(\omega-\omega_{0}\right)^{2}+\left\|\boldsymbol{X}-\boldsymbol{X}_{0}\right\|^{2}}
$$

Where an unknown frequency $\omega$ is introduced. The variables $\omega_{0}$ and $\boldsymbol{X}_{0}$ are the last converged solutions and the radius is $d s$. Let $\boldsymbol{Y}=\left\{\omega, \boldsymbol{X}^{T}\right\}^{T}$ denote the new unknown vector. The step by step descriptions of the arc-length solver for the multi-harmonic balance equation are as follows:

1). Obtain $m$ initial solutions of the equation in weak nonlinear region by Newton-Raphson iteration and compute arc-length control variable $s_{q}(q=2, \ldots, m)$ of these $m$ solutions, and set $s_{1}=0$.

$$
s_{q}=\sum_{p=1}^{q-1}\left\|\boldsymbol{Y}_{p+1}-\boldsymbol{Y}_{p}\right\|
$$

2). Start outer iteration $(j=m+1, m+2, m+3, \ldots)$. Compute new arc-length control variable $s_{\mathrm{j}}$

$$
s_{j}=s_{j-1}+d s
$$

3). Start inner iteration $(k=1,2,3, \ldots)$. The Lagrange extrapolation predictor [7] based on the last $m$ computed solutions is thus used to initialize the vector $\boldsymbol{Y}$.

$$
\boldsymbol{Y}_{j}^{(1)}=\sum_{p=j-m}^{j-1} \prod_{\substack{q=j-m \\ q \neq p}}^{j-1}\left(\frac{s_{j}-s_{q}}{s_{p}-s_{q}}\right) \boldsymbol{Y}_{p}
$$

4). Compute the Jacobian matrix $\boldsymbol{J}$ of the nonlinear system [8] at $\boldsymbol{Y}_{j}^{(k)}$.

$$
\boldsymbol{J}=\left[\begin{array}{cc}
\frac{\left(\omega_{j}^{(k-1)}-\omega_{j-1}\right)}{\left\|\boldsymbol{Y}_{j}^{(k-1)}-\boldsymbol{Y}_{j-1}\right\|} & \frac{\left(\boldsymbol{X}_{j}^{(k-1)}-\boldsymbol{X}_{j-1}\right)^{T}}{\left\|\boldsymbol{Y}_{j}^{(k-1)}-\boldsymbol{Y}_{j-1}\right\|} \\
\frac{\partial \boldsymbol{A}}{\partial \omega} \boldsymbol{X}_{j}^{(k-1)}-\frac{\partial \boldsymbol{F n}}{\partial \omega} & \boldsymbol{A}-\frac{\partial \boldsymbol{F n}}{\partial \boldsymbol{X}}
\end{array}\right]
$$

5). Compute the residual vector $\boldsymbol{R}=\left\{\boldsymbol{R}_{1}, \boldsymbol{R}_{2}^{T}\right\}^{T}$. If $\|\boldsymbol{R}\|<$ err, then stop inner iteration, let $j=j+1$, go to step 2); else go to step 6).

$$
\left\{\begin{array}{c}
\boldsymbol{R}_{1}=\left\|\boldsymbol{Y}_{j}^{(k-1)}\right\|-d s \\
\boldsymbol{R}_{2}=\boldsymbol{A} \boldsymbol{X}_{j}^{(k-1)}-\boldsymbol{F n} \boldsymbol{n}^{(k-1)}-\boldsymbol{F e}
\end{array}\right.
$$

6). Solve the linear equation.

$$
\left\{\Delta \omega, \Delta \boldsymbol{X}^{T}\right\}^{T}=\boldsymbol{J}^{-1} \cdot \boldsymbol{R}
$$

7). Update $\boldsymbol{Y}_{j}^{(k)}$, let $k=k+1$, go back to step 4).

$$
\boldsymbol{Y}_{j}^{(k)}=\boldsymbol{Y}_{j}^{(k-1)}-\left\{\Delta \omega, \Delta \boldsymbol{X}^{T}\right\}^{T}
$$

\section{Dry friction model}

The non-linear restoring force between the contacting surfaces of a bladed-disk system, such as root-disk joints or shrouds, are usually modelled by means of an elastic Coulomb friction model [5]. It should be noticed that, due to vibration, the normal load $N(t)$ is inevitably nonconstant in practical bladed-disks. So during relative motion of the surfaces three states are possible: stick, slip or separation.

$$
f_{n}=\left\{\begin{array}{cc}
f_{0}+k_{x} \cdot\left(x-x_{0}\right) & \text { stick } \\
\xi_{0} \mu N(t) & \text { slip } \\
0 & \text { seperation }
\end{array}\right.
$$

Where $\mu$ and $k_{x}$ are the friction and stiffness coefficient of the Coulomb model. $\xi_{0}$ is a sign function of the friction force at the time instant of slip state initiation; $x_{0}$ and $f_{0}$ are values of the relative tangential displacement and the interaction force at the beginning of the stick state. However, the constants $\xi_{0}, x_{0}$ and $f_{0}$ are history-dependent, and the transition states for the periodic set should be analysed in this model [9].

The Jacobi matrix in Eq. (12) of this friction model can be calculated by DFT in the time-domain, as shown in Eq. (18), where $g(t)$ is the harmonic basis function. It should be noticed that the force harmonic coefficient $\boldsymbol{F} \boldsymbol{n}$ not only depends on the relative displacement $x$ but also $x_{0}$ and $f_{0}$. The detailed derivation process can be seen in [2], which has been proved can overcome difficulties in convergence of the Newton-Raphson method.

$$
\frac{\partial \boldsymbol{F} \boldsymbol{n}}{\partial \boldsymbol{X}}=\frac{2}{T} \int_{0}^{T}\left(\frac{\partial \boldsymbol{f} \boldsymbol{n}}{\partial x} \frac{\partial x}{\partial \boldsymbol{X}}+\frac{\partial \boldsymbol{f} \boldsymbol{n}}{\partial x_{0}} \frac{\partial x_{0}}{\partial \boldsymbol{X}}+\frac{\partial \boldsymbol{f} \boldsymbol{n}}{\partial f_{0}} \frac{\partial f_{0}}{\partial \boldsymbol{X}}\right) \cdot g(t) \mathrm{d} t
$$




\section{Lumped parameter model for bladed disk}

In this paper, a one-dimensional lumped parameter model (LPM) has been devised to study the phenomena of a bladed disk system. The high-fidelity model consists of 30 symmetric sectors as shown in the left Fig. 1. Each sector is simplified as 4 DOFs, including the blade tip, middle root and disk. Two adjacent sectors are coupled by the disk DOFs and the boundary conditions are considered by the stiffness and damping of ground. All the values of structural parameters calculated according to actual dimensions and material properties of bladed disk are reported in Table 1 .

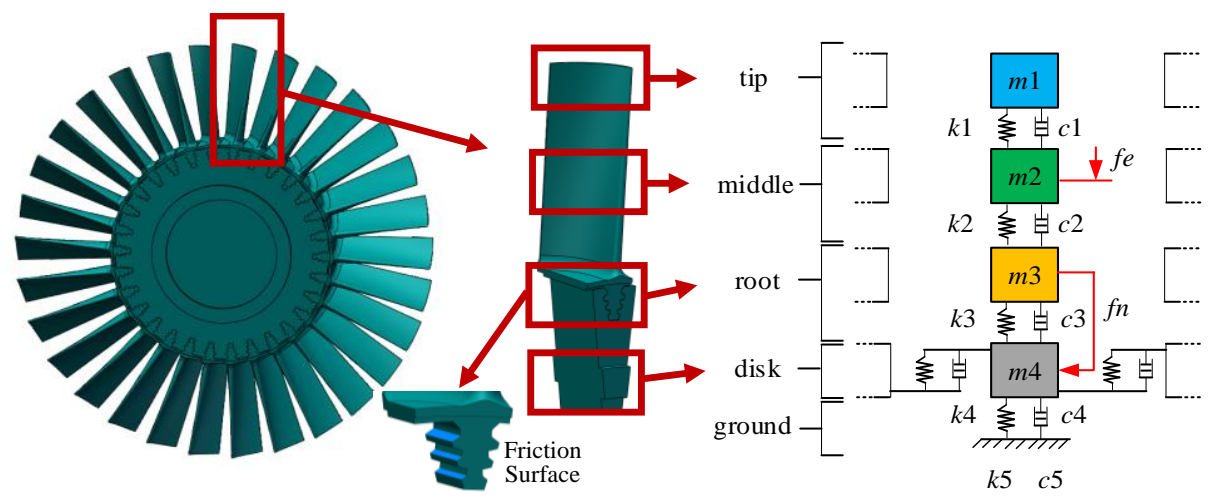

Fig 1. LPM for the bladed disk with dry friction nonlinearity

Table 1. Parameters values of LPM.

\begin{tabular}{|c|c|c|c|c|c|}
\hline $\begin{array}{c}\text { Parameter } \\
\text { (unit) }\end{array}$ & Tip & Middle & Root & Disk & Ground \\
\hline $\mathrm{m}(\mathrm{kg})$ & 0.02 & 0.1 & 0.055 & 0.1 & - \\
\hline $\mathrm{c}(\mathrm{N} \bullet \mathrm{s} / \mathrm{m})$ & 0.4 & 0.4 & 0.4 & 0.4 & 0.4 \\
\hline $\mathrm{k}(\mathrm{N} / \mathrm{m})$ & $5 \times 10^{5}$ & $2 \times 10^{6}$ & $1 \times 10^{8}$ & $5 \times 10^{7}$ & $10^{7}$ \\
\hline
\end{tabular}

In a turbomachine, the external excitation acted on the blade disk is mainly gas exciting force, which can be simplified to a circular periodic excitation. Here the excitation force is expressed as a cosine traveling wave with one nodal diameter $f e(t)=E_{0} \cos (\omega t+2 \pi j / N b)$, while the force is applied on the middle of $j^{\text {th }}$ blade as shown in Fig. 1. The nonlinear dry friction force $f n$ is applied on the disk and the root of the blade to simulate the coupling of two surfaces, which is the function of the relative displacement between the disk and root DOFs. The parameters $k_{x}$ and $\mu$ of the Coulomb friction model is set

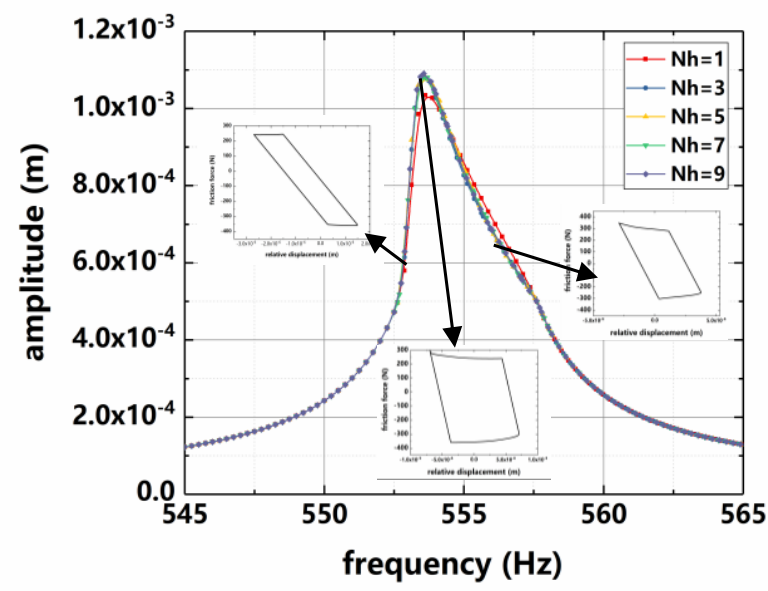

to $2 \times 10^{8}$ and 0.3 respectively. The normal $N(t)$ load force between the contacting surfaces is also regarded as one nodal diameter wave expressed as Eq. (19).

$$
N(t)=N_{0}+A_{0} \cos \left(\omega t+\frac{2 \pi j}{N b}\right)
$$

\section{Results and discussion}

The vibration characteristics of the LPM bladed disk with different normal loads $N_{0}$ and $A_{0}$ are presented and discussed in this section. The residual condition in step 5) err $=10^{-9}$ is used to check the convergence of the iteration process. The natural frequencies of the first family modes of the linear system are calculated for two special cases: bonded and frictionless interfaces of the root-disk, while frequencies of first nodal diameter are $555.13 \mathrm{~Hz}$ and $552.37 \mathrm{~Hz}$ respectively.

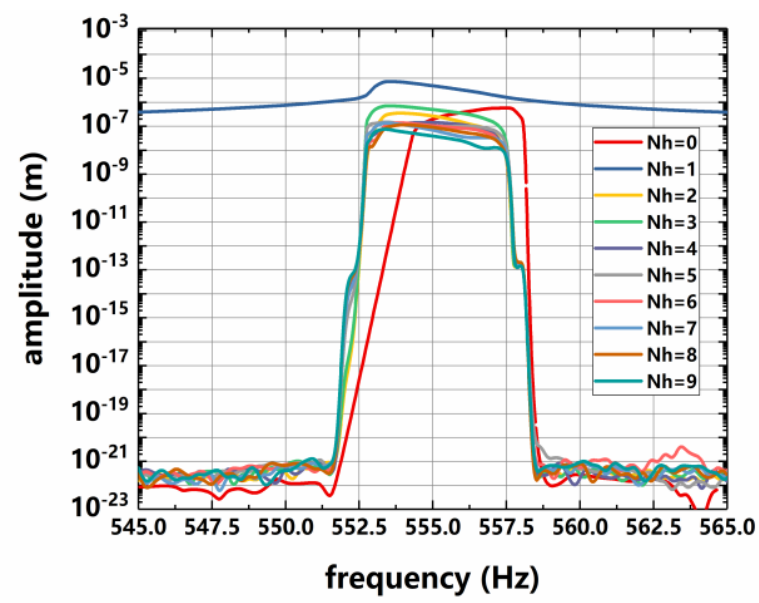

Fig. 2. Influence of the number of different retained harmonics on the accuracy of the solution 
First of all, the forced response with different numbers $N h$ of the retained harmonics curves are compared. As suggested in [6], a sufficient number of harmonics should be kept to account for the stick-slip transitions occurring at the interface when the nonlinearity is activated. It can be clearly seen that as $N h$ increasing from 1 to 9 , the response curves can converge gradually, and the hysteresis loops illustrate that the stick-slip transitions can be described accurately. It should be pointed out that, more accurate than approximate linear model, which is equivalent to the single harmonic method $(N h=1)$, the responses near resonance frequency are obviously higher. Further, the harmonic spectrum of the multi-harmonic vibration is shown quantitatively in the right side of Fig. 2. To reconcile the computational cost and the accuracy, 7 harmonics are kept in this study.

In this section, the excitation amplitude $E_{0}$ is set to $5 \mathrm{~N}$, with an excitation direction perpendicular to the blade surface. Different normal loads ranging from $600 \mathrm{~N}$ to $3000 \mathrm{~N}$ are selected to be the contact pressure between

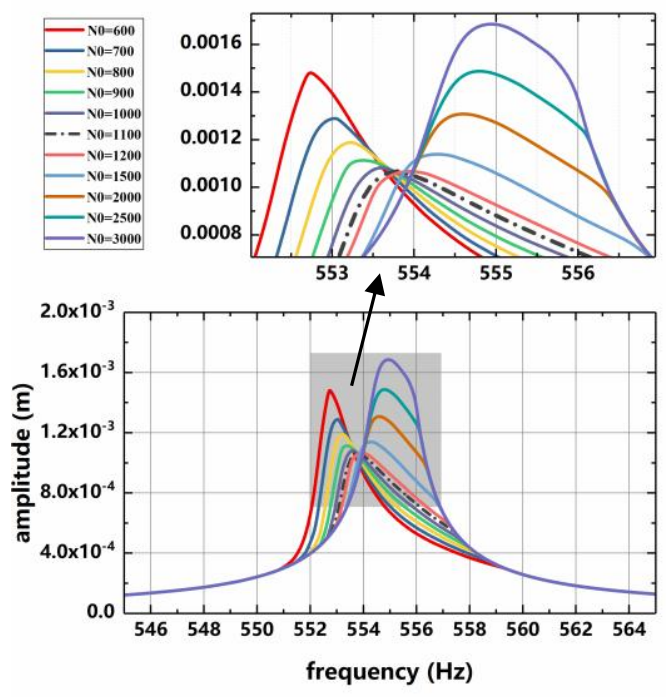

interfaces, as shown in Fig. 3. It can be seen that, there is an optimal load at $1100 \mathrm{~N}$ (dotted line), which makes the forced vibration response amplitude at the blade tip to be a minimum. The effect of dry friction damping is obvious that the maximal vibration amplitude of whole circle blades under the normal load of $1100 \mathrm{~N}$ is $0.107 \mathrm{~mm}$. However, if the normal load becomes an inappropriate value, which means the contact interfaces of root-disk joints are too loose or tight, the response may improve significantly, especially the normal load is designed for a smaller value.

The resonance frequency is equal to that of the underlying linear system $(555.13 \mathrm{~Hz})$ with bonded DOFs at the interface, which means the contact interfaces is too tight. Once the normal load decreases to certain extent, the friction interface steps into slip state completely and the resonance frequency will be close to frictionless linear model $(552.37 \mathrm{~Hz})$. The phenomenon reveals that the resonance frequency gradually shifts from the bonded model toward the frictionless model.

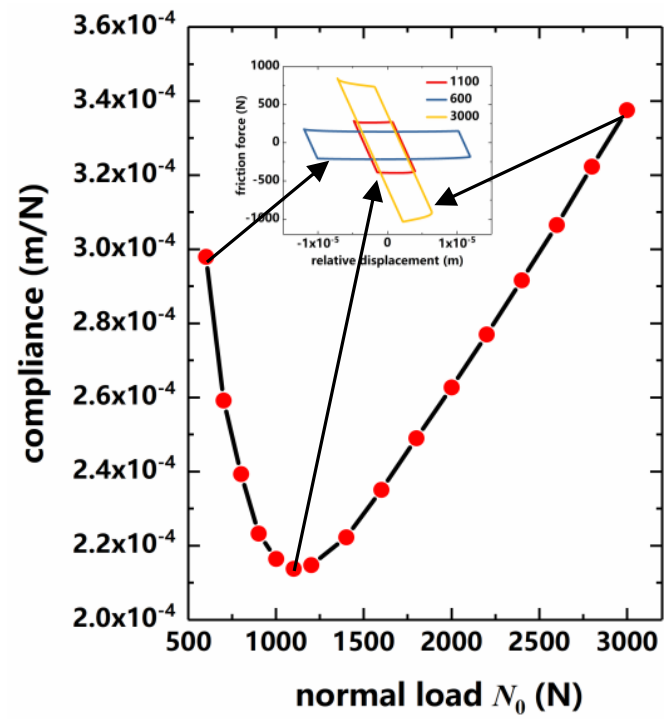

Fig. 3. Response amplitude curves under different normal loads $N_{0}$

Further, the compliance of the blade tip at the resonant peak frequency with different normal loads is given by the right graph in Fig. 3, which can be expressed as the ratio of the maximum displacement amplitude to the excitation level $E_{0}$. It can be observed clearly that with an optimum in terms of damping for a normal load $N_{0}$ of about $1100 \mathrm{~N}$ noticeable in the graph. Meanwhile, due to the nonlinear friction damping characteristics, hysteresis curves of dry friction damped could be influenced significantly by the magnitude of normal load under the same excitation, which lead to resonance amplitude and resonance frequency out of original state.

These results indicate that for a specific blade disk structure, there exist a suitable contact normal load design under the excitation force of general operating conditions. This analysis method proposed in this paper can accurately predict this optimal normal load. In this numerical example, the optimal displacement amplitude of the blade tip can be reduced by $28.26 \%$ compared with that of normal load at $600 \mathrm{~N}$, which means better vibration performance of the bladed-disk.

Last but not least, the sensitive of normal load at the optimal value is investigated, with the normal load fluctuation $A_{0}$ varying from 0 to $1000 \mathrm{~N}$. It should be emphasized that the contact surfaces have no separation during the periodic time. The hysteresis loop of the friction surface degenerates into the form of general Coulomb friction for $A_{0}=0 \mathrm{~N}$, which is a parallelogram. As the $A_{0}$ increases, the hysteresis loop develops into a closed curve, meanwhile the relative displacement between the contact surfaces expands slowly. However, the incensement is so small that the vibration amplitude of blade tip has no much difference (from $0.106 \mathrm{~mm}$ to $0.119 \mathrm{~mm}$ ) as shown in Fig. 4. It appears that the vibration performance of system is insensitive to variation of normal load fluctuations.

a Corresponding author: tianyuannn@163.com 

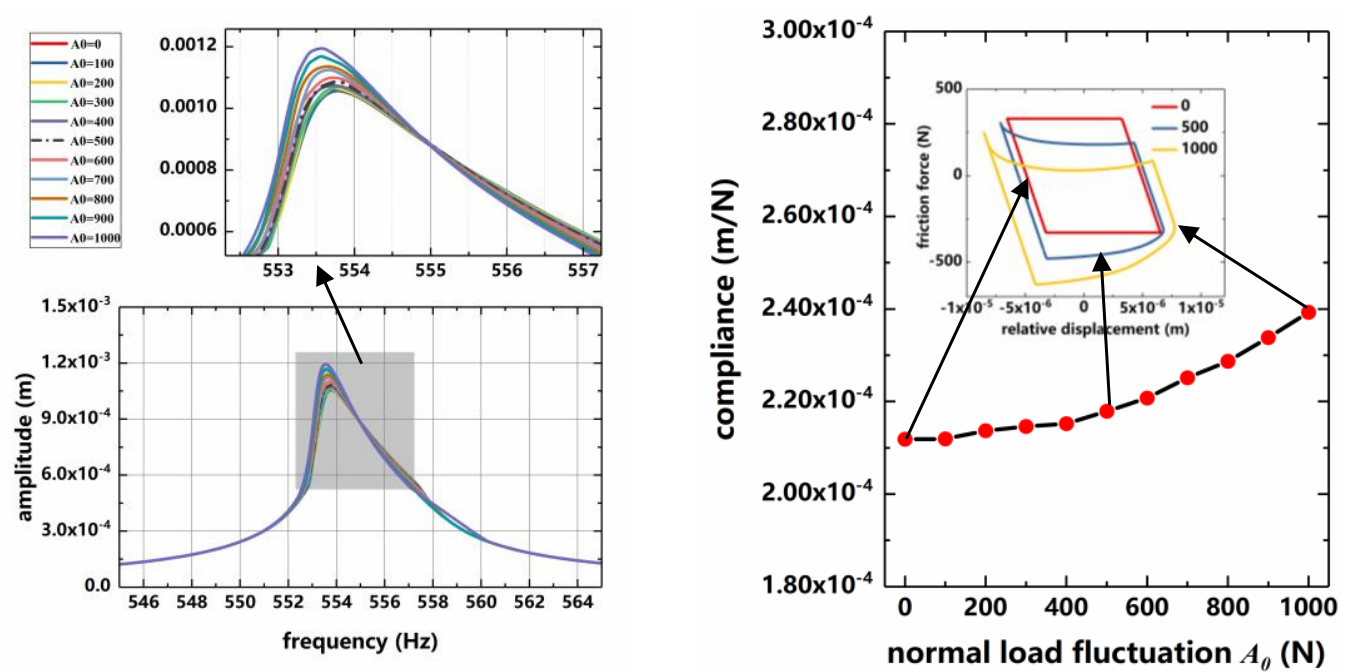

Fig. 4. Response amplitude curves under different normal load fluctuations $A_{0}$

\section{Conclusions}

This paper focuses on the vibration characteristics of the bladed-disk subjected to the dry friction damping under periodic excitation. A lumped parameter model is applied to investigate the influence of normal load of the blade root-disks on the vibration response. Firstly, the forced response with different numbers of the retained harmonics curves are compared, which reveals that the approximate linear model shows poor accuracy in the resonance region. Then the forced response with different normal loads are performed to seek an optimal norm load for the bladed-disk design. It appears that the resonance frequency gradually shifts from the tight normal load to the loos normal load and the optimal value can be obtained by the qualitative analysis of the nonlinear vibration characteristic. At last, the influence of the normal load fluctuations for the friction interfaces has also been addressed. The results show that the vibration performance of system is insensitive to variation of normal load fluctuations, which can be a supplement to the robustness evaluation at the optimal normal load of the bladed-disk design. The investigation provides a qualitative assessment for the design practice of friction dampers.

\section{References}

1. Jun W, Ruishan Y, Zhenwu H, et al. Experimental Study on Dry Friction Damping Characteristics of the Steam Turbine Blade Material with Nonconforming Contacts[J]. Advances in Materials Science \& Engineering, 2015, 2015: 1-15.

2. Petrov E, Ewins D. Analytical Formulation of Friction Interface Elements for Analysis of Nonlinear Multi-Harmonic Vibrations of Bladed Discs[J]. Journal of Turbomachinery, 2003, 125(2):364-371.

3. Petrov, E, and Ewins, D, 2006, Effects of Damping and Varying Contact Area at Bladed-Disk Joints in Forced Responses Analysis of Bladed Disk
Assemblies, ASME J Turbomach., 128(2), pp. 403410.

4. Petrov, E., Zachariadis, Z., and Beretta, A., 2012, A Study of Nonlinear Vibrations in a Frictionally Damped Turbine Bladed Disk with Comprehensive Modeling of Aerodynamic Effects, ASME J. Gas Turbines Power, Vol.3, pp. 1239-1251.

5. Laxalde, D, and Thouverez, F, 2009, "Complex NonLinear Modal Analysis for Mechanical Systems: Application to Turbomachinery Bladings with Friction Interfaces, Journal of Sound Vibration $[\mathrm{J}]$, 322(4-5), pp. 1009-1025.

6. Joannin, C., Chouvion, B., and Thouverez, F., 2015, Nonlinear modal analysis of mistuned periodic structures subjected to dry friction, ASME J. Eng. Gas Turbines Power[J], Vol.7

7. Cheung $\mathrm{Y}$ K. Application of the incremental harmonic balance method to cubic non-linearity systems. Journal of Sound \& Vibration[J], 1990, Vol. 140, No 2, pp. 273-286.

8. Ferreira J V, Serpa A L. Application of the arclength method in nonlinear frequency response". Journal of Sound \& Vibration[J], 2005, Vol. 284, No 1, pp. 133-149.

9. Yang, B.D., Chu, M.I. and Menq, C.H., Stick-slipseparation analysis and non-linear stiffness and damping characterization of friction contacts having variable normal load, Journal of Sound \& Vibration, Vol. 210, No 4, 1998, pp. 461-481.

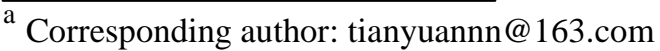

